\title{
Condiciones de trabajo con respecto a la salud ocupacional de las empleadas de higienización de la UNAH
}

\author{
Yolanda Argentina Molina Fonseca' \\ Ana Corina Hernández Rodríguez ${ }^{2}$
}

\section{RESUMEN}

El propósito de este artículo es dar a conocer los resultados que se obtuvieron en la investigación realizada sobre la salud ocupacional de las empleadas de la Universidad Nacional Autónoma de Honduras (UNAH), cuyo objetivo general fue analizar las condiciones de trabajo con respecto a la salud ocupacional de las empleadas de higienización de la UNAH.

En el estudio se caracterizaron las condiciones en que desempeñan su labor las empleadas de higienización de la UNAH y resultados obtenidos reflejan que estas condiciones representan riesgos por accidentes o enfermedades profesionales. Asimismo, se elaboró un perfil actual de salud de la población estudiada, en el cual prevalecen las enfermedades músculo-esqueléticas, alergias respiratorias y dermatológicas.

La metodología utilizada para llevar a cabo la investigación fue de tipo cualitativa. La selección de las unidades primarias a quienes se les hizo la entrevista a profundidad fue intencionada al momento de hacer las agrupaciones de acuerdo al año de ingreso a laborar en la institución y de forma aleatoria se seleccionó a una persona por agrupación.

En respuesta a esta problemática, el estudio identificó algunas acciones de protección, tales como el uso de guantes y mascarillas, aunque este último implemento no lo usan con la regularidad necesaria. Las acciones de reacción encontradas en el estudio son: reducción de las actividades laborales, cambio de actividades laborales y traslados a otros departamentos en la misma jornada de trabajo.

Palabras claves: condiciones de trabajo, salud laboral, políticas, acciones de prevención, acciones de protección y acciones de reacción.

\footnotetext{
Beneficiario de una beca básica de la DICYP. Estudiante, Posgrado Latinoamericano en Trabajo Social con Orientación en Gestión del Desarrollo, Departamento de Trabajo Social, Facultad de Ciencias Sociales, UNAH:yolanda.molina@unah.edu.hn

${ }^{2}$ Asesora, Facultad de Ciencias Sociales, docente del Postgrado Latinoamericano en Trabajo Social y de la Carrera de Trabajo Social: platsunah@yahoo.es
} 


\section{ABSTRACT}

The purpose of this article is to present the results obtained in the research on occupational health of the employees of the National Autonomous University of Honduras (UNAH). The overall objective of this research is to analyze the conditions of work for occupational health of the employees UNAH sanitation.

In the study the conditions under which the employees perform their work sanitizing UNAH characterized, results show that these conditions pose risks for accidents or occupational diseases.

An actual health profile of the population studied, which are prevalent musculoskeletal, respiratory and skin diseases was developed allergies.

The study identified some protective actions such as gloves and masks, although the latter do not use attachment with the regularity needed. Actions of reaction found in the study are: reduction of work activities, change of work activities, transfers to other departments in the same working day.

Keyworks: working conditions, healthy labor, politics, prevention actions, protection actions, reaction actions.

\section{INTRODUCCIÓN}

La salud ocupacional es un tema considerado como parte de las políticas sociales que garantizan el bienestar de los hondureños que prestan sus servicios a la empresa privada, instituciones gubernamentales o realizan trabajos independientes. Al respecto, la Secretaría de Trabajo y Previsión Social, la Secretaría de Salud y el Instituto Hondureño de Seguridad Social, han realizado acciones encaminadas a proteger la salud de los trabajadores hondureños, pero las mismas no logran dar respuesta para evitar los riesgos por accidentes 0 enfermedades profesionales.

El Departamento de Servicios Generales de la UNAH, ubicado en la Ciudad Universitaria, es el espacio laboral de los sujetos del presente estudio. Este Departamento cuenta con aproximadamente 580 empleados que se distribuyen en siete (7) secciones: albañilería, carpintería, electricidad, fontanería, jardinería, higienización y soldadura. ${ }^{3}$

\footnotetext{
${ }^{3}$ Información proporcionada por el personal del Depto. de Servicios Generales, mayo de 2012.
} 
La sección de higienización tiene la responsabilidad de recolectar los desechos sólidos, limpiar las áreas verdes y los edificios. Las personas que forman parte del estudio, están dedicadas particularmente a la limpieza de edificios y áreas verdes.

La presente investigación se hizo con el propósito de conocer las condiciones de trabajo de los empleados de higienización de la Universidad Nacional Autónoma de Honduras (UNAH), en la Ciudad Universitaria, su situación de salud y cómo las acciones de prevención, de protección para evitar alteraciones en la salud al realizar su trabajo y las acciones de reacción que implementa la UNAH ante la presencia de enfermedades ocupacionales.

La pregunta que orientó esta investigación fue cómo son las condiciones de trabajo con respecto a la salud ocupacional de las empresas de higienización de la Universidad Nacional Autónoma de Honduras, por lo cual se plantearon los objetivos siguientes:

\section{Objetivo general}

Analizar las condiciones de trabajo de los empleados de higienización de la Universidad Nacional Autónoma de Honduras.

\section{Objetivos específicos}

1. Caracterizar las condiciones en que desempeñan su labor los empleados de higienización de la UNAH.

2. Elaborar un perfil de salud de los empleados de aseo de la UNAH.

3. Identificar las acciones de prevención, protección y reacción dirigidas a los empleados de aseo de la UNAH.

\section{METODOLOGÍA}

La metodología utilizada para llevar a cabo la investigación fue de tipo cualitativa. La selección de las unidades primarias a quienes se les hizo la entrevista a profundidad fue intencionada al momento de hacer las agrupaciones de acuerdo al año de ingreso a laborar en la institución y de forma aleatoria se seleccionó a una persona por agrupación.

\section{Procedimiento metodológico}

1. Se aplicaron siete (7) entrevistas a profundidad dirigidas a las aseadoras de la institución. Para seleccionar la muestra hizo una agrupación de los empleados 
por quinquenio a partir de 1981 a 2013, habiendo obtenido 7 quinquenios, se seleccionó aleatoriamente a una persona por quinquenio.

2. Se realizaron nueve (9) entrevistas semiestructuradas así: 4 al personal de supervisión de higienización, 2 a los miembros de la Comisión de Higiene y Seguridad, 2 a las autoridades universitarias y 1 a las autoridades sindicales. Los informantes claves que fueron seleccionados están directamente involucrados en el tema y toman decisiones en la institución.

El análisis de datos se realizó a través de la comparación de la información obtenida, tanto de las unidades de análisis primarias como de los informantes claves, para lo cual, una vez recopilada la información, se clasificó y organizó; posteriormente se vació en dos matrices previamente elaboradas, una para las unidades de análisis primarias y otra para los informantes claves.

\section{ANÁLISIS}

Los hallazgos del proceso de la investigación se agrupan en seis aspectos que se describen a continuación:

\section{Situación laboral actual}

Las labores que desempeñan en la institución es la limpieza de mobiliario y equipo, aseo de oficinas, aulas, pasillos, corredores, gradas, sanitarios, paredes, techos internos, puertas y ventanas. Para realizar estas labores utilizan escobas, recogedores de basura, trapeadores, mechas, espátulas para sacar la cera de chicles de los pisos y sillas, baldes, pastes, sacudidores, pailas y bolsas para basura.

Las aseadoras dicen que la Universidad les proporciona los materiales para realizar su trabajo, pero que los mismos son de baja calidad y limitados, ya que tienen que solicitar con anticipación a la fecha programada de entrega de materiales, que es cada 15 días, y en algunas ocasiones tienen que comprar de su dinero las bolsas para basura.

2. Manejo de químicos

Se realizan prácticas inapropiadas en el manejo de químicos, puesto que deben traer el recipiente para que se les den los químicos que vienen en líquido. El personal del Departamento de Servicios Generales confirmó la 
información proporcionada por las entrevistadas, pues este Departamento hace entrega de los químicos a las supervisoras, quienes los distribuyen a las aseadoras en botellas transparentes de 1.5 litros, sin rotular.

La Universidad no cuenta con un equipo multidisciplinario de profesionales de las áreas de medicina, microbiología, química, ingeniería, psicología y trabajo social que realice las estrategias y lineamientos para implementar medidas de prevención y protección, lo cual trae como consecuencia que las condiciones de trabajo que tienen actualmente las mujeres de higienización incremente el riesgo en la alteración de su salud.

Anteriormente, el Departamento de Servicios Generales proporcionaba al personal de aseo el desmanchador para los sanitarios, pero este químico fue sustituido por el cloro, porque el desmanchador alteraba la salud de las aseadoras; así lo expresó el subjefe de este Departamento.

3. Condiciones de espacio físico

Las aseadoras y el personal de supervisión manifiestan que el espacio físico donde están ubicados los cuartos de aseo no reúne las condiciones mínimas de iluminación, ventilación y amplitud, además de la inseguridad que hay, porque en la mayor parte de los cuartos de aseo están ubicadas las cajas de control de electricidad.

Los espacios físicos destinados para el personal de aseo miden $6.4 \mathrm{mts}^{2}$, lo que indica una capacidad para dos personas, pero en la práctica se alojan hasta ocho aseadoras; ahí toman sus alimentos, sirven de bodega de materiales y químicos, y en algunos casos el cuarto de aseo está en los baños, en otros no tienen tomacorriente para que ellas puedan calentar sus alimentos.

4. Medidas de prevención, protección y reacción de la UNAH para las empleadas de higienización

A través de la investigación se logró identificar las acciones de prevención, protección y reacción que la UNAH realiza para evitar que la salud de las empleadas de aseo se altere al realizar las labores asignadas.

\section{Medidas de prevención}

Las aseadoras y el personal de supervisión manifiestan que hasta el momento no han recibido capacitación sobre medidas de prevención y protección al 
realizar su trabajo. Las capacitaciones recibidas han sido orientadas solamente para la de limpieza.

\section{Medidas de protección}

Según las aseadoras, la institución les proporciona guantes y mascarilla como implementos de protección y manifiestan que los utilizan, aunque no lo hacen con la frecuencia necesaria para protegerse de riesgos; las mascarillas por lo general no las usan y los guantes los usan para lavar los sanitarios, recoger la basura e ir a depositarla a los contenedores.

\section{Medidas de reacción}

Sobre las acciones de reacción que la institución realiza se registra que, una vez que la aseadora presenta la certificación médica, temporalmente previamente a una reubicación, se le reduce el área de trabajo o se cambia de labor; por ejemplo, los casos de las personas que padecen de lumbalgias, a estas las ubican en el aseo de sanitarios para evitar que levanten mobiliario, así lo reporta el Departamento de Servicios Generales.

Al analizar las condiciones en que laboran las empleadas de aseo de la UNAH, se considera que las mismas pueden influir en la alteración de la salud de las mujeres aseadoras, por las actividades repetitivas, por el levantamiento constante de mobiliario y equipo, por la exposición a productos químicos. Todo esto puede causar enfermedades musculoesqueléticas, dermatológicas y afecciones de las vías respiratorias.

5. Perfil de salud de las empleadas en el área de higienización

Según la información proporcionada por las aseadoras y el personal de supervisión, entre los accidentes de trabajo más frecuentes están el deslizamiento cuando están lavando, golpes en las manos y pies cuando tienen que mover y levantar mobiliario pesado (escritorios, archivos, mesas) y accidentes de tránsito al trasladarse de su lugar de residencia a su lugar de trabajo o viceversa; pero este último no lo consideraba como accidente de trabajo.

Con respecto a la situación de salud de las aseadoras, la enfermedad más frecuente que se observa en las entrevistadas es la presión alta, la cual se manifiesta con vértigo y mareo, por lo que algunas asisten al Instituto 
Hondureño de Seguridad Social (IHSS) para su control y tratamiento; en estos casos, el IHSS recomienda no subir gradas, no agitarse mucho y evitar los ruidos. Sin embargo, otras no se están controlando la presión arterial. Otro de los problemas de salud que reportan son los dolores lumbares y musculares y el estrés.

Las incapacidades extendidas han sido por maternidad, por fractura de pie y extirpación de quiste; las hospitalizaciones han sido por maternidad y por extirpación de quiste.

Actualmente en la institución existe la unidad de Salud Ocupacional adscrita a la Secretaría Ejecutiva de Desarrollo de Personal. Esta analiza la situación de salud del personal de aseo y reporta que del total de las 269 aseadoras, 34 tienen constancia médica porque presentan alteraciones en su salud, las más comunes son las lumbalgias, afecciones respiratorias y afecciones de la piel.

El Departamento de Servicios Generales también conoce de la situación de salud de las empleadas de higienización y reporta que los problemas lumbares son los más frecuentes.

6. Tensiones con relación a la prevención y protección de la salud de las mujeres de higienización

Según las autoridades universitarias, hay un rechazo por parte de las aseadoras para usar en debida forma los implementos de protección, pues ellas no tienen conciencia del peligro que representa el no uso de los implementos, ya que no han recibido charlas sobre medidas de prevención y protección. Además, la institución no tiene un reglamento que obligue a este personal para que realice sus labores considerando las medidas de prevención y protección.

Por su parte, las autoridades sindicales confirman que hasta la fecha no ha habido una reglamentación en la cual los trabajadores asuman la responsabilidad de tomar las medidas de protección al realizar sus labores. La organización sindical en la negociación de los contratos colectivos se ha dedicado a obtener derechos.

La situación de salud de las empleadas de aseo que laboran en la UNAH demanda de la atención inmediata tanto por parte de las autoridades universitarias como de las autoridades sindicales, pues las estadísticas así lo reflejan. 


\section{DISCUSIÓN}

La revisión de investigaciones relacionadas con la temática de la salud ocupacional sirvió de referente para la definición del tema de estudio. En la presente investigación se logró conocer cómo son las condiciones laborales y la situación de salud de las aseadoras de la UNAH. Para desarrollar este tema se utilizó de referencia a algunos autores como la OMS (2008), quien establece que la salud y la enfermedad de las personas es el resultado de muchos factores que inciden significativamente en el proceso que se da entre la vida y la muerte de cada persona en una sociedad determinada.

La teoría de la multicausalidad define la salud como: "El resultado observable en las y los individuos de la comunidad, producto de la relación existente entre los medios disponibles y los factores de riesgo que la amenazan en un momento histórico determinado" (OMS, 2008).

Leavell y Clark, probablemente sean los precursores de la teoría de la causalidad. La Corporación para Investigaciones Biológicas (2005), los cita al plantear los tres niveles de prevención, los cuales se describen a continuación.

La prevención primaria está referida a promocionar y proteger la salud de las enfermedades, donde se pretende que las personas mantengan un estado de normalidad de bienestar físico y mental en equilibrio con su ambiente, para lo cual recomiendan, por un lado, la realización de exámenes periódicos e higiene; y por el otro, hablan de la prevención específica en la cual se tomen medidas para cada enfermedad y para prevenir accidentes laborales.

La prevención secundaria se realiza a través del diagnóstico precoz, donde se detectan los factores de riesgo en los primeros estadios y dar tratamiento oportuno buscando la limitación del daño de una enfermedad en evolución, tratando de retrasar los deterioros y erradicar las causas.

La prevención terciaria es el proceso de rehabilitación física, socioeconómica y psicológica. Para corregir hasta donde sea posible la incapacidad o el daño producido y para que pueda adaptarse la persona a sus condiciones de vida y fomentar la productividad.

Tomando como base los resultados obtenidos en la investigación, se puede decir que tanto las condiciones laborales, la manipulación de químicos y no tomar las medidas preventivas y de protección necesarias, son factores que pueden alterar la salud de las aseadoras al realizar sus labores en la institución. 


\section{CONCLUSIONES}

Al realizar la caracterización de las condiciones en que desempeñan su labor las empleadas de higienización de la Universidad Nacional Autónoma de Honduras, se pudo constatar que hay problemas, es decir, que no son idóneas para un nivel óptimo de salud, por lo que representan riesgos por accidentes o enfermedades profesionales. Las entrevistadas reportan que las condiciones que la institución tiene no son las más adecuadas, con lo que se corrobora lo que la OMS (2008) plantea, acerca de que si el trabajo no es adecuado, gratificante y dignificante, puede agredir a quien lo realiza hasta llegar a enfermarle.

Otro aspecto relevante que se observó al hacer la investigación es que en la institución no hay una política sobre salud ocupacional de los trabajadores universitarios, aunque se han hecho acciones conjuntas con las autoridades sindicales; pero estas acciones se enfocan más en medidas de reacción y no de prevención, lo cual indica que al no haber medidas de prevención existe un alto riesgo de alterar la salud de las empleadas de aseo de la institución, agudizado por la poca conciencia de parte de estas empleadas al no tomar las medidas de protección al momento de realizar su trabajo (usar guantes, mascarillas, zapato cerrado o botas para lavar los edificios).

La prevención supera a la reacción, porque al prevenir, como lo plantean Leavell y Clark citados por la Corporación para Investigaciones Biológicas (2005), se pretende que las personas mantengan un estado de normalidad de bienestar físico y mental en equilibrio con su ambiente, de tal forma que se logre evitar llegar al accidente o la enfermedad.

En consecuencia, es importante destacar que existe una comisión de higiene y seguridad bipartita UNAH-SITRAUNAH, la misma no está siendo reconocida por las autoridades universitarias, debido al conflicto interno que existe en la organización sindical; entonces, se evidencia lo que Foucault (1979) plantea sobre el poder, sobre que este se orienta hacia la dominación, las formas de sometimiento y los dispositivos de estrategia, de tal forma que esta comisión no ha tenido la oportunidad de planificar estrategias orientadas a la prevención de accidentes y enfermedades profesionales.

La comisión cuenta con un reglamento que regula su actuar, pero no existe un reglamento interno que obligue a las empleadas de higienización a tomar las medidas de protección necesarias para realizar su labor, únicamente se les ha permitido desempeñar acciones aisladas como la entrega de uniformes, botiquines 
con medicamentos y conocer algunas situaciones de salud para tratar de reubicar a las empleadas.

La situación es más crítica cuando las autoridades universitarias y sindicales no tienen un plan de acción orientado a concienciar a las empleadas a través de capacitaciones y campañas sobre la prevención de accidentes y enfermedades ocupacionales, por lo que se ven limitadas a autoprotegerse de estos riesgos.

Una vez que la enfermedad aparece, la reubicación de las empleadas de aseo se ve limitada por el nivel de escolaridad que tienen algunas de ellas, cuya recomendación médica es el cambio de actividades laborales; esto se agudiza más cuando las alternativas no son muchas, pues como lo plantea Amorós (1995), las oportunidades de empleo de las mujeres están limitadas por los estereotipos y normas sociales.

El espacio físico asignado al personal de aseo es inadecuado y, en algunos casos, con alto riesgo de sufrir accidentes, ya que es el mismo espacio físico donde están ubicadas las cajas de control de energía eléctrica. También, hay deficiencia en cuando a la amplitud, ventilación e iluminación de estos espacios.

\section{AGRADECIMIENTO}

A la Universidad Nacional Autónoma de Honduras por haberme otorgado una beca para realizar esta investigación a través de la Dirección de Investigación Científica y Posgrados.

\section{BIBLIOGRAFÍA}

Amorós, Celia. (1995). Palabras y conceptos clave en el vocabulario de la igualdad. Mujeres en red. El periódico feminista. Recuperado de: http://www.mujeresenred.net/spip.php?article1301

Carmenate Milián, L. (2011). Perfil nacional seguridad y salud en el trabajo (SST). Tegucigalpa: Programa Salud y Trabajo en América Central.

Cobo Bedia, R. (1995). 10 palabras clave sobre mujer. Valencia: Editorial Verbo Divino.

CODEMUH y Universidad Autónoma Metropolitana de Xochimilco. (2012). Condiciones de trabajo y prevalencia de trastornos musculoesqueléticos y psíquicos en población trabajadora de la maquila de la confección. Honduras: REDCAM.

Colectivo de Mujeres de Honduras. (2006). Situación de las mujeres de la maquila en Honduras. Trabajo y salud. Tegucigalpa. 
Escuela de Salud Pública de la Universidad de Griffith, Australia. (2003). De una promoción de la salud en el lugar de trabajo hacia la gestión integradora de la salud en el lugar de trabajo: tendencias y evolución. Recuperado de: www.who.int/occupational_health/publications/newsletter/en/gohnet6s.pdf.

Red Mundial de la Salud Ocupacional. (2003). De una promoción de la salud en el lugar de trabajo hacia la gestión integradora de la salud en el lugar de trabajo: tendencias y evolución. The Global Ocupational Healh Network, 6, 1-4.

Foucault, M. (1979). Microfísica del poder. Madrid: Las Ediciones de La Piqueta.

IHSS. (2011). Guía metodológica de prevención de riesgos laborales. Tegucigalpa.

IHSS. (2012). Evento día mundial de la salud y seguridad en el trabajo. Tegucigalpa.

Instituto Hondureño de Seguridad Social. (2012). IHSSS en cifras serie 2003-2011. Recuperado de: www.ihss.hn.

OIT. (s.f.). Memorando técnico: diagnóstico de la inspección del trabajo en Honduras. Recuperado de: http://www.ilo.org/wcmsp5/groups/public/--ed_dialogue/---lab_admin/documents/publication/wcms_119252.pdf.

Oliva, X. F. (2010). Los factores de riesgo que enfrentan las empleadas que manipulan los desechos bioinfecciosos en el edificio de Ciencias BiológicasCiudad Universitaria de la Universidad Nacional Autónoma de Honduras (UNAH), durante el año de 2010. Tegucigalpa.

PNUD. (s.f.). Informe de Desarrollo Humano 2008. Tegucigalpa. 